
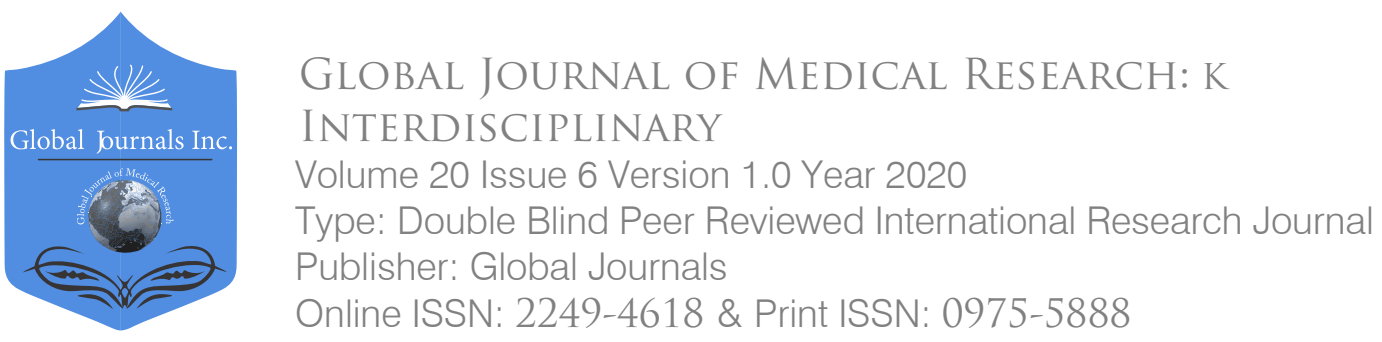

\title{
Results of the Olfactory Cognition Test Performed on 45 Female University Students
}

By Naomi Katayama, Shoko Kondo, Chika Aoki, Ai Kagazume, Mari Tashita

\& Kasumi Yano

Nagoya Women's University

Abstract- The sense of smell is related to the quality of life and not only can protect oneself from dangers such as food poisoning, gas leakage, and fire. But also various pleasures such as enjoying a delicious meal and the scent of flowers. This time, we report that we conducted an olfactory cognitive test using open essence on healthy 45 female university students. The Open Essence (made by FUJIFILM) has the smell as same as the odor Stick Identification Test (OSIT-J). The aromas used in the open essence includes curry, perfume, Japanese cypress, India ink, menthol, rose, wood, stinky socks/sweat, roasted garlic, condensed milk, gas for cooking, and Japanese mandarin aromas. This 12 different odorants perception is not necessarily culture-free; the Japanese version employed. Depending on the type of odor, some of them were difficult to understand, and some that were easy to understand. The most willrecognized odor was the smell of stinky socks/sweat, and the most hard to understand odor was mandarin orange. In the future, it is necessary to individually examine not only the number recognized in the olfactory recognition test but also the odor that was understand.

Keywords: olfaction test, open essence, cognition, female, university student.

GJMR-K Classification: NLMC Code: W 925

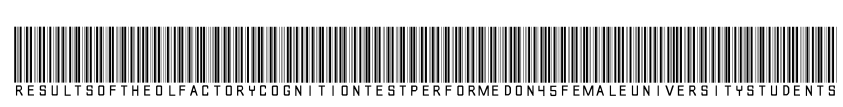

Strictly as per the compliance and regulations of:

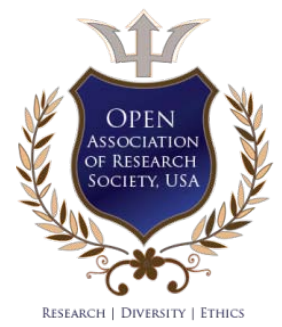

(C) 2020. Naomi Katayama, Shoko Kondo, Chika Aoki, Ai Kagazume, Mari Tashita \& Kasumi Yano. This is a research/review paper, distributed under the terms of the Creative Commons Attribution-Noncommercial 3.0 Unported License http://creativecommons.org/licenses/by-nc/3.0/), permitting all non-commercial use, distribution, and reproduction in any medium, provided the original work is properly cited. 


\title{
Results of the Olfactory Cognition Test Performed on 45 Female University Students
}

\author{
Naomi Katayama ${ }^{\alpha}$, Shoko Kondo ${ }^{\circ}$, Chika Aoki $^{\rho}$, Ai Kagazume ${ }^{\omega}$, Mari Tashita ${ }^{\ddagger}$ \& Kasumi Yano ${ }^{\S}$
}

Abstract-The sense of smell is related to the quality of life and not only can protect oneself from dangers such as food poisoning, gas leakage, and fire. But also various pleasures such as enjoying a delicious meal and the scent of flowers. This time, we report that we conducted an olfactory cognitive test using open essence on healthy 45 female university students. The Open Essence (made by FUJIFILM) has the smell as same as the odor Stick Identification Test (OSIT-J). The aromas used in the open essence includes curry, perfume, Japanese cypress, India ink, menthol, rose, wood, stinky socks/sweat, roasted garlic, condensed milk, gas for cooking, and Japanese mandarin aromas. This 12 different odorants perception is not necessarily culture-free; the Japanese version employed. Depending on the type of odor, some of them were difficult to understand, and some that were easy to understand. The most will-recognized odor was the smell of stinky socks/sweat, and the most hard to understand odor was mandarin orange. In the future, it is necessary to individually examine not only the number recognized in the olfactory recognition test but also the odor that was understand.

Keywords: olfaction test, open essence, cognition, female, university student.

\section{INTRODUCTION}

$\mathrm{n}$ recent years, research on olfactory disorders has attracted attention as an early symptom of COVID-19 and Alzheimer's dementia. The olfactory disorder refers to a state in which an odor cannot felt tolerance, and the most common cause is nasal sinus disease. There is a part called the olfactory mucosa in the part of nose that corresponds to the ceiling of the left and right nasal cavities. When an odorous substance in the air adheres to this olfactory mucosa, a stimulus signal transmitted to the brain through the olfactory nerve, and it feels as an odor. Olfactory disorders classified into 1) Respiratory, 2) Peripheral, 3) Central, depending on where the odor transmission pathway is impaired. Corona virus, which is currently a problem, is a peripheral olfactory disorder, and olfactory mucosal olfactory disorder in which the olfactory mucosa degenerates is suspected. Alzheimer's olfactory disorder is a central olfactory disorder in which the brain that processes odor information is damaged.

Author $\alpha \rho \omega ¥ \S$ : Nagoya Women’s University, Nagoya City, Japan. e-mail:naomik@nagoya-wu.ac.jp

Author a: Graduate School of Nagoya Women's University, Nagoya City, Japan.

Author $\sigma:$ Watanabe Hospital, Mihama town, Noma, Aichi, Japan.
Therefore, this study focused on the perception of smell and aimed to understand the actual situation of 12 different kinds of odors closely related to Japanese life in each age and gender. To begin with, we report on the olfactory perception of the young females.

\section{il. Materials and Methods}

\section{a) Participants}

The participants were female students $(n=45)$ who voluntarily participated in olfactory tests. Females students were third-year, fourth-year, and graduate students. Age \pm standard deviation was $20.42 \pm 0.50$ years old. The maximum was 21 years old, and the minimum was 20 years old. They were healthy, not going to the hospital and taking no medication. They were self-reported and had no colds and no fever.

\section{b) Assessment of odor identification}

The Odor Stick Identification Test (OSIT-J) was used to assess odor perception for many years for our study. This test possesses high reliability and validity 1 ). The commonly used procedure resembles that of the San Diego Odor Identification Test 2). The aromas used in the OSIT-J include curry, perfume, Japanese cypress, India ink, menthol, rose, wood, stinky socks/sweat, roasted garlic, condensed milk, gas for cooking, and Japanese mandarin aromas3,4).. This 12 different odorants perception is not necessarily culture-free; the Japanese version was employed 3,4).. Each fragrance was enclosed in microcapsules made of melamine resin3, 4).

In this study, we use The Open Essence (made by FUJIFILM) has the smell as same as the odor Stick Identification Test (OSIT-J). The open essence is a card type, and the scent had already applied to the card. When participants open the card, it has the same scent as OSIT-J. Each correct answer was scored as one point with the total performance score ranging from 0 to 12 points 5). We defined it as follows: normal range as more than 6 points, borderline as 3 to 5 points, and abnormal as less than 2 points 5). All of these methods are the same as in the previously reported paper 5). The results are that the perceivable odor was the average on the12 types of $8.5 \pm 1.7$.

c) Ethical review board

This study conducted with the approval of the Ethical Review Board (Nagoya women's university Ethics 
Committee: 'hitowomochiitakennkyuunikansuruiinnkai'). The approval number is 30-11.

\section{Results}

a) Odor identification (number of the correct answers)

Twelve different kinds of olfactory cognitive tests conducted on female students by using the Open Essence. The results shown in Table 1. When there are six or more types of recognition among the 12 types of odors, it is considered as an acceptable range (we call it a normal range). This time, 44 out of 45 female students could recognize more than six kinds of odors. By the way, one student had four types of perceptible odors. The average value of the olfactory recognition test results of 45 female university students was $8.5 \pm 1.7$ (Table1. and Table 2.).

Table 1: Results of olfactory cognition test using open essence in female university student $(n=45)$

\begin{tabular}{lccccccccccccc}
\hline \hline & 0 & 1 & 2 & 3 & 4 & 5 & 6 & 7 & 8 & 9 & 10 & 11 & 12 \\
\hline Open essence (number of student) & 0 & 0 & 0 & 0 & 1 & 0 & 4 & 10 & 5 & 11 & 10 & 3 & 1 \\
Open essence (\%) & 0.0 & 0.0 & 0.0 & 0.0 & 2.2 & 0.0 & 8.9 & 22.2 & 11.1 & 24.4 & 22.2 & 6.7 & 2.2 \\
\hline \hline
\end{tabular}

Table 2: Results of olfactory cognition test using open essence in female university student (Average number of recongnition \pm Standard Deviation)

\begin{tabular}{lc}
\hline & Numver of recognition \pm Standard Deviation \\
\hline Open essence $(n=45)$ & $8.5 \pm 1.7$ \\
\hline \hline
\end{tabular}

b) Odor identification (percentage of each smell)

Next, Table 2 shows the results of individually examining each of the 12 odors. This scent of India ink is the odor that we have always smelled when we calligraphy in elementary school. However, the correct answer rate was as low as $51.1 \%$. The smell of wood had a correct answer rate of $71.1 \%$. Some students dislike perfume, and the correct answer rate was $55.6 \%$. Menthol was a poultice scent, and the correct answer rate was $93.3 \%$, which was very high. The scent of mandarin orange seemed to be very incomprehensible, with the lowest correct answer rate being $28.9 \%$. The

scent of curry was $97.8 \%$, probably because it is a familiar odor in daily life. The correct answer rate for household gas was $86.7 \%$, which was high. The scent of rose is the scent that female prefer, but the correct answer rate was $82.8 \%$. Cypress is a wood that often used for furniture in Japan, and the correct answer rate for this scent was $80.0 \%$. All the 45 female students recognized the smell were stinky socks/sweaty odors. The correct answer rate of condensed milk was $68.9 \%$. The odor of stir-fried garlic had a correct answer rate of $33.3 \%$, the second-lowest next to mandarin oranges (Table 3.)

Table 3: Results of olfactory cognition test for 12 different odors in female university students using open essence $(n=45)$

\begin{tabular}{|c|c|c|c|c|c|c|c|c|c|c|c|c|}
\hline & Indea Ink & Wood & Perfume & Menthol & Mandarin orange & Curry & Household gas & Rose & Cypress & Stinky socks/Sweaty & Indensed m & Stirffried garlic \\
\hline Open essence (number of student) & 23.0 & 32.0 & 25.0 & 42.0 & 13.0 & 44.0 & 39.0 & 37.0 & 36.0 & 45.0 & 31.0 & 15.0 \\
\hline Open essence $(\%)$ & 51.1 & 71.1 & 55.6 & 93.3 & 28.9 & 97.8 & 86.7 & 82.2 & 80.0 & 100.0 & 68.9 & 33.3 \\
\hline
\end{tabular}

\section{Discussion}

An olfactory cognition test was performed using open essence on 45 healthy female university students who did not have a cold, did not have a fever, and did not take any medicine. The perceivable odor was the average on the12 types of $8.5 \pm 1.7$. Since they were healthy in their twenties, their olfactory cognitive ability was high. However, looking at the 12 odors individually, there were odors with a high percentage of correct answers and low odors. And the odor of mandarin orange and the odor of stir-fried garlic had low accuracy. It found that these two kinds of odors are difficult to understand even though they are female university students, although they are the odors that they come into contact with every time they eat. However, almost everyone knew the smell of curry. Curry is a familiar scent that young females often eats. Most female students could also recognize the smell of stinky socks/ sweat and the smell of household gas. Since the odor of socks is a rotten odor, understanding this odor helps prevent food poisoning. Also, understanding the smell of house hold a gas helps prevent gas explosion and gas poisoning. The authors performed olfactory cognition tests using open essence in the same way as this time on the elderly in the past (Naomi katayama 2020). As a result, in the case of older adults, the number of odors recognized by the olfactory recognition test was about 6-7 on average. It can see that the results of the olfactory cognition test of young female university students are well. In the future, we could like to obtain the results of olfactory cognition tests for 
students in their teens and middle-aged age and compare them by age group.

\section{Conclusions}

An olfactory cognitive test performed on young female university students in their 20's using open essence. As a result, out of the 12 odors, $8.5 \pm 1.7$ odors were recognized on average. This value is higher than that of the elderly score, and the odor is well known. Depending on the type of odor, that was difficult to understand, and some that were easy to understand. In the future, it is necessary to individually examine not only the number recognized in the olfactory recognition test but also the odor that recognized. Furthermore, we think that we can understand the age-related changes in olfactory cognition by examining the changes with each age.

\section{Acknowledgements}

This study was supported by the research aid of Choju-iryo-kenkyu-kaihatsuhi 30-14 and the Japanese Society of Taste Technology, 2019.

\section{References Références Referencias}

1. Kobayashi M (2005). The odor Stick Identification Test for the Japanese (OSIT-J): Clinical suitability for patients suffering from olfactory disturbance. Chemical Senses, 30(Suppl 1): i216-i217.

2. Murphy C, Anderson AJ, Markinson S (1994). Psychophysical assessment of chemosensory disorders in clinical populations. In K. Kurihara, N. Suzuki, \& H. Ogawa (Eds.) Olfaction and Taste. Tokyo: Springer Verlag Tokyo. pp. 609-613.

3. Kobayashi M, Reiter ER, DiNardo JL, Costanzo MR (2007). A new clinical olfactory function test: cultural influence. Arch. Otolaryngol-Head Neck Surg. 133(4): 331-336.

4. Kobayashi M, Saito S, Kobayakawa T, Deguchi Y, Costanzo RM (2006). Cross-cultural comparison of data using the Odor Stick Identification Test for Japanese (OSIT-J). Chem. Senses. 31(4): 335-342.

5. Katayama N, Kondo S, Ootake H et al (2018). Odour and Salt Taste Identification in Older Adults: Evidence from the Yakumo Study in August, 2018. Acade. J. Med. Plants 7(3) 066-071.

6. Naomi Katayama, Shoko Kondo, Satofumi Sugimoto, Tadao Yoshida, Masaaki Teranishi, Michihiko Sone3 Yasushi Fujimoto, Hironao Otake, Hirokazu Suzuki, Takafumi Nakada, Naoki Saji, Seiichi Nakata and Tsutomu Nakashima (2019. Odour and salt taste identification in older adults: Evidence from the Yakumo. Academia Journal of Medicinal Plant, 8(3): 030-035. 